\title{
A Simulation Model Validation and Calibration Platform
}

\author{
Shenglin Lin, Wei Li, Xiaochao Qian, Ping Ma, Ming Yang* \\ Control and Simulation Center, Harbin Institute of Technology, China \\ lin_44627079@yeah.net, frank@hit.edu.cn, everqxc@ hotmail.com, pingma@ hit.edu.cn,myang@hit.edu.cn
}

\begin{abstract}
The simulation model validation and calibration (SMVC) is a complicated work, including uncertainty description, many simulation experiments execution and complex data analysis etc. Moreover, there are many uncertainty factors such as model form assumptions and solution approximations, random variability of model inputs, etc. need to be considered when requiring a precise model. For assisting the SMVC effectively, this paper develops a software platform to validate and calibrate the simulation models when some quantities may be affected by uncertainty. First, an unprecedented process model, which includes uncertainty description, simulation experiment design, model validation and model calibration, is presented to explicate the procedures of SMVC under uncertainty. In the process model, many new model validation and calibration algorithms under uncertainty are applied based on our previous work. Second, the design of platform is divided into two parts, which consist of structure design and function design, and the software technique based on strategy pattern is introduced to integrate and maintain the SMVC algorithms. Then this platform is implemented according to its expected uses and key design requirements. Finally, application example of model validation and calibration of a flight vehicle kinematic control system is illustrated how to use the platform.
\end{abstract}

Keywords: model validation, model calibration, uncertainty description, software platform

\section{Introduction}

Simulation models are increasingly used to solve practical problems in various engineering disciplines. The basic premise of the simulation-based solutions is the credible simulation models, so model validation is introduced naturally, which is used to validate the simulation models by measuring the extent of agreement between the model output and experimental observations (Sankararaman and Mahadevan, 2011). However, the credibility of simulation models are also affected by various sources of uncertainty such as model form assumptions and solution approximations, natural variability in model inputs and parameters, and data uncertainty due to sparse and imprecise information. For improving the precision of simulation models further, the uncertainty parameters must be described and determined. So model calibration is proposed to improve the quality of simulation models through adjusting model parameters according to the results of model validation. Besides, in order to research the SMVC under uncertainty better, the model uncertainties are classified according to their fundamental essence as either (a) aleatory - the inherent variation in a quantity that, given sufficient samples of the stochastic process, can be characterized via a probability density distribution, or (b) epistemic uncertainty due to lack of knowledge by the modelers, analysts conducting the analysis, or experimentalists involved in modeling and simulation (Roy and Oberkampf, 2011).

The simulation model validation and calibration (SMVC) process under uncertainty is a complicated and tedious work, which involves quantification of various uncertainties, design of many simulation experiments, complex data management and analysis, application of many kinds of SMVC methods, etc. Thus, an auxiliary platform is needed to develop for assisting the SMVC under uncertainty. Currently, the development of SMVC under uncertainty platform is still in the initial stage, such as some modeling and validation platforms are listed below. A comprehensive platform of modeling and simulation credibility evaluation is developed (Balci et al, 2002). An integrated simulation measurement platform is developed by Institute for Simulation and Training (IST). Besides, some tools about uncertainty quantification and analysis are exploited. For example, an uncertainty quantification and analysis tool is implemented for the automated operation of uncertainty quantification and analysis (Ferson, 2002). An automated tool about the probability boundary calculation interval-based is developed by Berleant (1993). Simlab is an integrated uncertainty and sensitivity analysis tool, which is used to solve the sample generation of uncertainty variables, uncertainty propagation and analysis (Saltelli et al, 2005).

The existing auxiliary platforms above cannot accomplish the SMVC under certainty completely and the integrated auxiliary platforms have not been researched. In order to assist to validate and calibrate simulation models, an integrated software platform, HIT-MVCP (Harbin Institute of Technology Model Validation and Calibration Platform), is designed and implemented, which can assist users to accomplish SMVC under uncertainty effectively. The remainder of 
this paper is organized as follows. A process model for SMVC under uncertainty is presented in Section 2. The structure and function of HIT-MVCP are designed in Section 3. Section 4 describes the software implementation of HIT-MVCP and an example of model validation and calibration of a flight vehicle kinematic control system is illustrated how to use the platform. Finally, the conclusion and the future work are summarized.

\section{The Process Model of SMVC under Uncertainty}

Due to the complex and tedious model validation and calibration procedures, a reasonable and explicit operation scheme is necessary before implementing SMVC under uncertainty. So a process model of SMVC under uncertainty is presented, which includes uncertainty description, experiment schemes design, simulation execution, model validation, consistency metamodeling, uncertainty parameters optimization (Figure 1). First, various uncertainty variables which impact the simulation execution should be determined and described primarily in special forms such as stochastic and interval variable (Helton, 2011). After that, for the subsequent model calibration according to the validation results, the propagation of the uncertainty effect from input information to simulation results needs to be researched (Helton et al, 2006) and the simulation experiment design is naturally used to solve this problem based on the design purposes and uncertainty expression. In the next step, the credibility of simulation model could be achieved through measuring the extent of agreement between the model output and experimental observations (Meng et al, 2015). Finally, for accomplishing model calibration, we merely need to adjust repeatedly the uncertainty parameters to maximize the data consistency. But, due to the effect of aleatory and epistemic uncertainty, the calibration process of uncertainty parameters needs plenty of experiment schemes design and simulation execution times and this will produce much computation cost. So a metamodel-based algorithm is applied to improve the efficiency of model calibration and reduce the computation expense. The specific description of the algorithm is researched in (Qian et al, 2016).

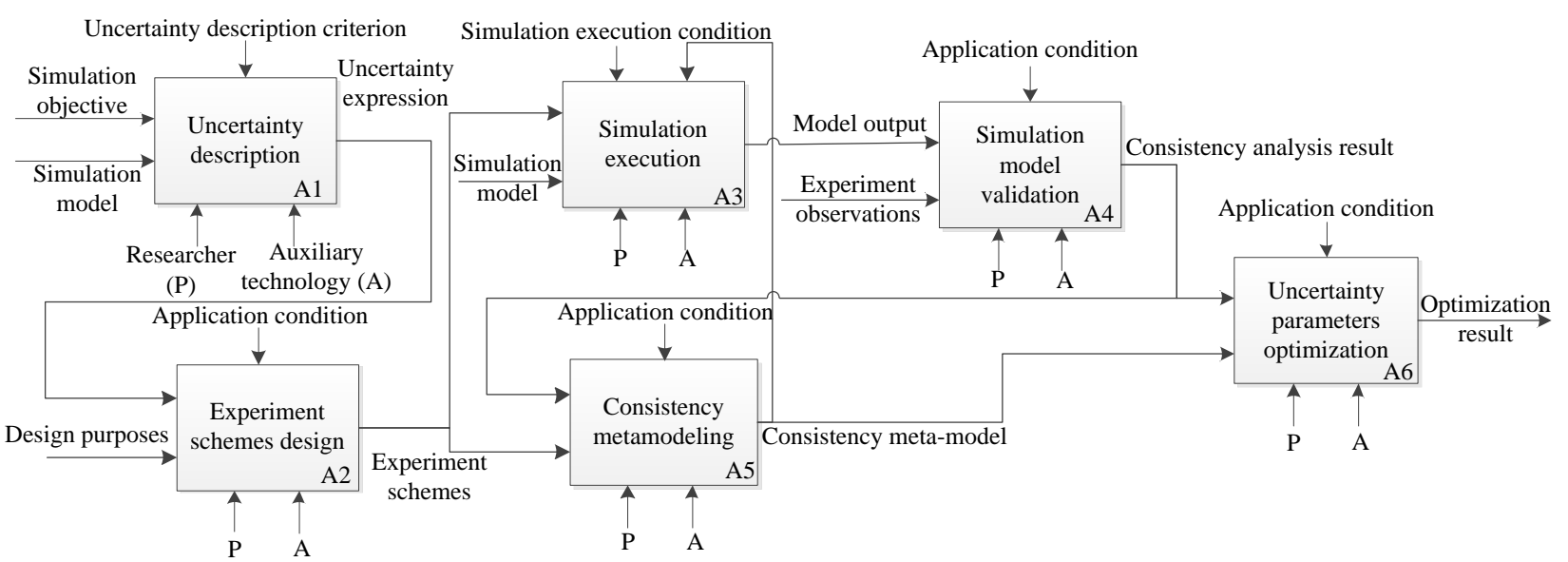

Figure 1. The SMVC process model.

The procedures of SMVC under uncertainty include following parts:

A. Uncertainty description (A1): According to the simulation objective, the uncertainty information involved in simulation execution would be described. The aleatory and epistemic uncertainties are expressed respectively in form of stochastic variable and interval variable.

B. Experiment schemes design and simulation execution (A2, A3): The uncertainty information is propagated from input parameters to simulation results based on the experiment design. The probability sampling methods are used such as, random sampling, LHS sampling, uniform sampling etc. and the experiment schemes could be stored and reused.

C. Simulation model validation (A4): We need to validate the extent of agreement between the model output and the experiment observations. These data with the simulation uncertainty often belong to different styles, such as dynamic data, static data etc. So, a validation method based evidence distance for multi-outputs under uncertainty is introduced (Qian et al, 2016) and the brief procedures are show as follows.

a. Extracting the data feature, such as shape, position, frequency spectrum etc. And constructing the feature matrix.

$b$. Evidence fusion. Transferring the feature matrix into form of evidence body.

c. The validation result could be achieved by means of computing the evidence distance among the evidence bodies.

D. Consistency metamodeling (A5): For reducing the 
computation expense, the consistency metamodel of multiple outputs is constructed. The simulation model calibration would be completed via model calibration and uncertainty parameters optimization. The epistemic uncertainty parameters are sampled by means of the experiment design and the simulation experimental outputs under each epistemic uncertainty sample are obtained. Each simulation result which only involves the aleatory uncertainty is used to make a consistency analysis with the reference data, and then the agreement metamodel with the aleatory uncertainty variable could be obtained.

E. Uncertainty parameters optimization (A6): The consistency metamodel is regarded as the objective function in the optimization process of epistemic uncertainty parameters and many optimization algorithms are used to achieve the appropriate parameter value. The procedures of simulation model calibration based on consistency metamodeling and parameters optimization are given as follows:

a. Designing and executing simulation experiment schemes to obtain the epistemic uncertainty samples and simulation results.

$b$. Evaluating the agreement degree between the model output and experiment observations.

c. According to the multiple epistemic uncertainty samples and the results of model validation, the consistency metamodel could be constructed.

d. To maximize the data consistency, the optimal epistemic uncertainty parameter could be determined.

\section{The Structure and Function Design of HIT-MVCP}

The proposed process model in Section 2 is regarded as the guideline of SMVC under uncertainty. According to the function description of each procedure in the process model, an auxiliary platform, HIT-MVCP, is designed which involves structure design and function design. However, many developing SMVC algorithms under uncertainty such as methods of the experiment scheme design, model validation algorithms, metamodeling methods etc. need to be integrated and maintained in the design process. If we maintain the platform continuously with the fast development of SMVC algorithms and this will generate high expense, and reduce the practicality of platform. So a software technique based on strategy pattern is introduced to design and implement the algorithms involved in SMVC process, and improve the maintainability and extensibility of HIT-MVCP.

\subsection{The Structural Design of HIT-MVCP}

Based on the functions and usage description of each procedure in the SMVC process model and modularized principle, the structure of HIT-MVCP is divided into five subsystems to respectively design, which including uncertainty description, experiment design and execution, simulation model validation, simulation model calibration, data management.

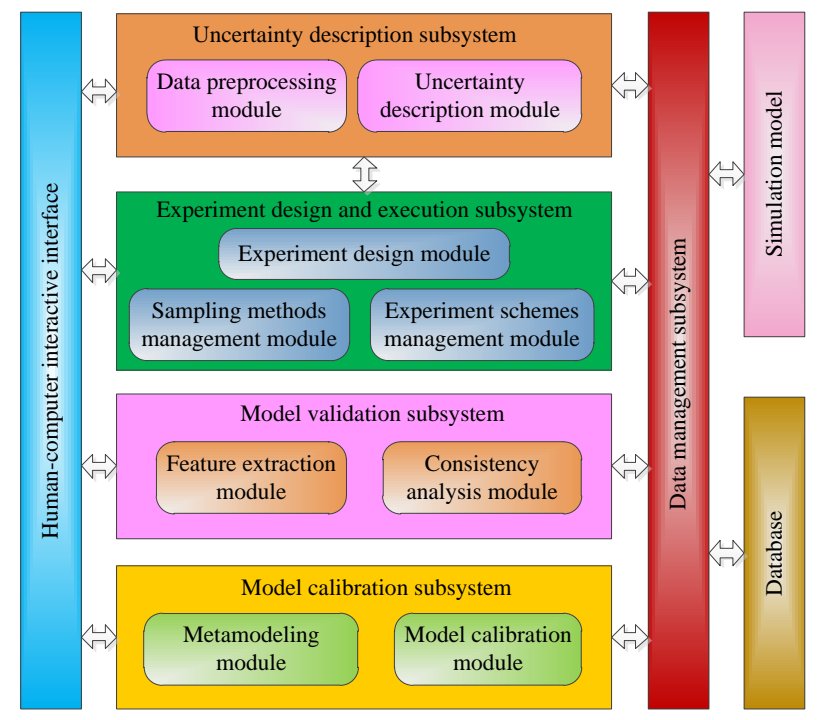

Figure 2. The structure design of HIT-MVCP.

The detailed design described in Figure 2 includes following subsystems:

A. Uncertainty description subsystem. In the subsystem of uncertainty description, we need to determine uncertainty factors of the simulation models and describe them in forms of probability based on the experiment observations and model output. Due to the uncertainty effect, there are some defects such as outliers etc. in simulation results. So the data preprocessing procedure need to be introduced and then the uncertainty variables can be expressed based on the data postprocessing.

$B$. Simulation experiment design and execution subsystem. This subsystem consists of three modules, which include experiment design, sampling methods management and experiment schemes management. The experiment design module is used to produce experiment schemes based on the sampling method from the module of sampling methods management. The experiment schemes are managed in experiment schemes management module and transmitted to data management subsystem. Then, the simulation model will receive and execute these experiment schemes.

C. Model validation subsystem. This subsystem is used to measure the degree of agreement between the model output and experimental observations. Based on the introduced model validation algorithm, the model validation subsystem is divided into two modules to design respectively, which include feature extraction and consistency analysis. And then the validation results will be stored in database through the data management 
subsystem.

D. Model calibration subsystem. Based on the presented calibration algorithm in Section 2, the model calibration subsystem is divided into metamodeling and model calibration. The consistency metamodel under aleatory uncertainty is constructed based on the experiment schemes and results of model validation. The consistency metamodel is regard as the objective function and the model calibration module is used to adjust epistemic uncertainty parameter based on many optimization algorithms.

E. Data management subsystem. This subsystem is responsible for providing the interaction interface between simulation model and databases. The information of SMVC process such as the storage and execution of experiment schemes, transmission of model output etc. are all managed via this subsystem.

With the development of SMVC techniques, many kinds of algorithms about SMVC under uncertainty are researched and applied such as random sampling, Kriging, Hypothesis testing etc. Assuming we integrate the new algorithms into HIT-MVCP constantly and the high cost and the bad practicability will be arisen. So the software technique of strategy pattern is used to design the related algorithms of SMVC under uncertainty and then the maintainability and extensibility of HIT-MVCP is improved effectively. Strategy pattern makes the alteration of algorithms independent from users through defining and encapsulating a family of algorithms which are interchangeable. The detail algorithm design based on strategy pattern is described in Section B.

According to the process model of SMVC under uncertainty and structure design, the related algorithms of HIT-MVCP are divided into eight types: data preprocessing, uncertainties described, sampling methods, feature extraction, validation principle, calibration principle, optimization methods and metamodeing (Figure 3). These algorithms are used in different phases of SMVC under uncertainty and the extended interface is design based on strategy pattern.

\subsection{The Structural Design of HIT-MVCP}

Depending on the process model of SMVC under uncertainty, HIT-MVCP is developed to realize two functions: model validation and model calibration. Model validation is used to measure the extent of agreement between model output and experiment observations. Based on the algorithm description of model validation and strategy pattern, model validation function is divided into two parts to design respectively, which including feature extraction and consistency analysis and the class diagram of software design is given in Figure 4. As the core of model validation subsystem, the model validation class relies on the data management subsystem, and helps coordinate the feature extraction and consistency analysis. The interaction procedure of each class is given in Figure 5.

Model calibration is used to determine the epistemic uncertainty of model for maximizing the degree of agreement between model output and experimental observations. According to the algorithm description of model calibration and strategy pattern in Section 2, this subsystem is divided into two modules to design respectively, which includes metamodeling and model calibration. The metamodeling module includes sequential design criterion and metamodeling type class, and completes the metamodeling based on sequential design. The model calibration subsystem involves optimization methods class and calibration criterion class, and is responsible for adjusting the epistemic uncertainty parameter. The class diagram of model calibration subsystem is given in Figure 6. As the core of the subsystem, the model calibration class is used for the data exchange and control, and depends on the data management subsystem to call the experiment schemes. The interaction procedure of each class is given in Figure 7.

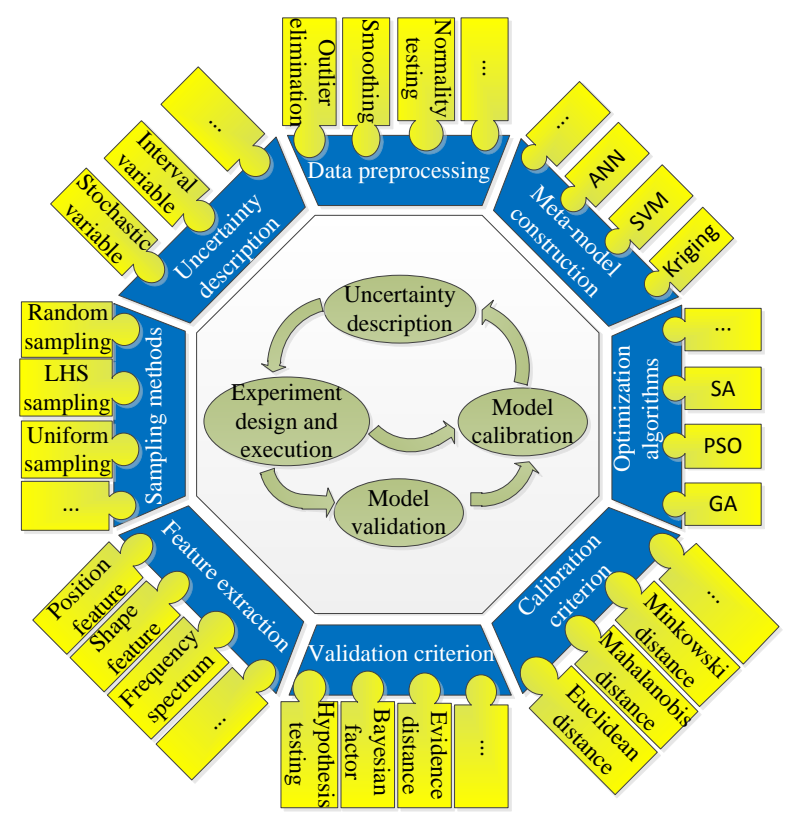

Figure 3. The algorithm structure design.

\section{The Implementation and Application of HIT-MVCP}

HIT-MVCP is implemented based on C++ language depending on the structure and function design above. The human-computer interaction interface of platform is given in Figure 8 and the all subsystems could be called from this interface. In addition, an example about the model validation and calibration of a flight vehicle kinematic control system is given to validate the efficiency of the described functions and illustrate how to use HIT-MVCP. This kinematic control model is crucial for movement simulation of a flight vehicle. 
Multiple disturbances and uncertainties will be encountered in simulation phase, and impact the control accuracy of flight vehicle such as initial mass and velocity of flight vehicle, atmospheric density, damping factor, etc. For making the terminal guidance simulation model more precise, the disturbances and uncertainties affecting terminal guidance model need to be described accurately, and researched how to influence the model prediction. Besides, for evaluating whether the model could reach the application standard of simulation and application, the model credibility under multiple uncertainties must be validated.

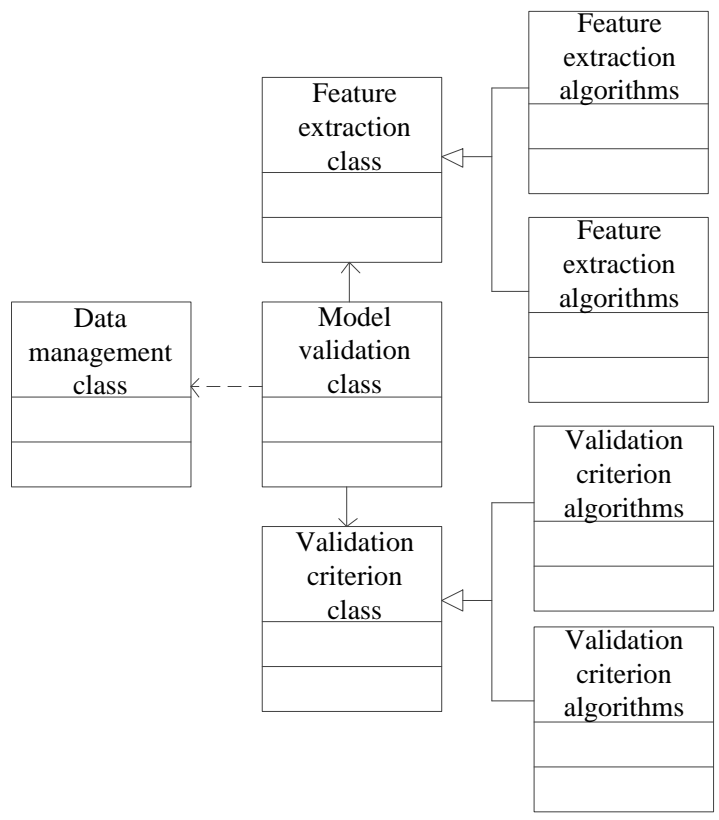

Figure 4. Class diagram of the model validation subsystem.

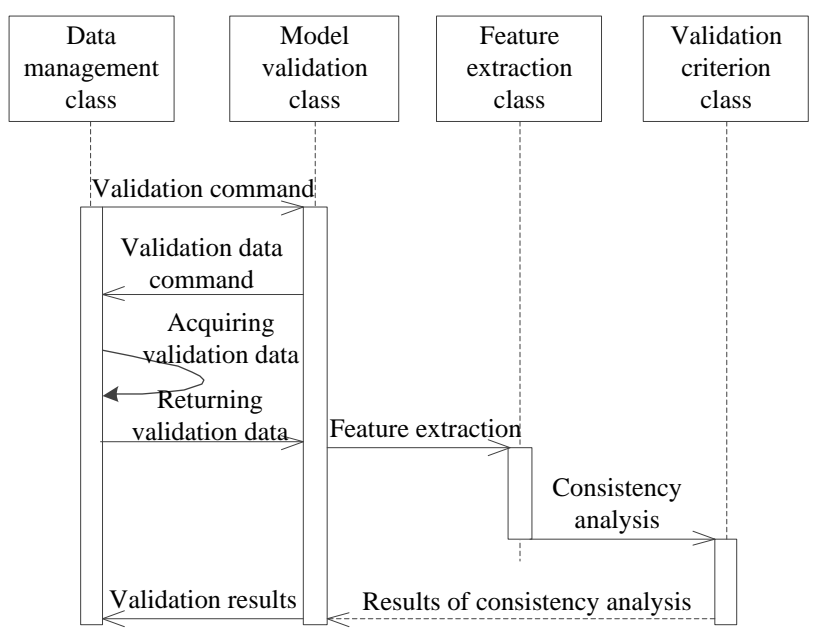

Figure 5. Interactive procedure of model validation.

According to the process model of SMVC under uncertainty, the uncertainty parameters involved in the simulation model firstly need to be described. Supposing the aleatory uncertainties consist of atmospheric density, initial velocity and angle of the flight vehicle, lift coefficient, damping factor and the epistemic uncertainties involve initial mass and reference area of the fight vehicle. The expression manner of the uncertainty parameters could be determined by means of parameter estimation of multiple sample data and user input directly (Figure 9).

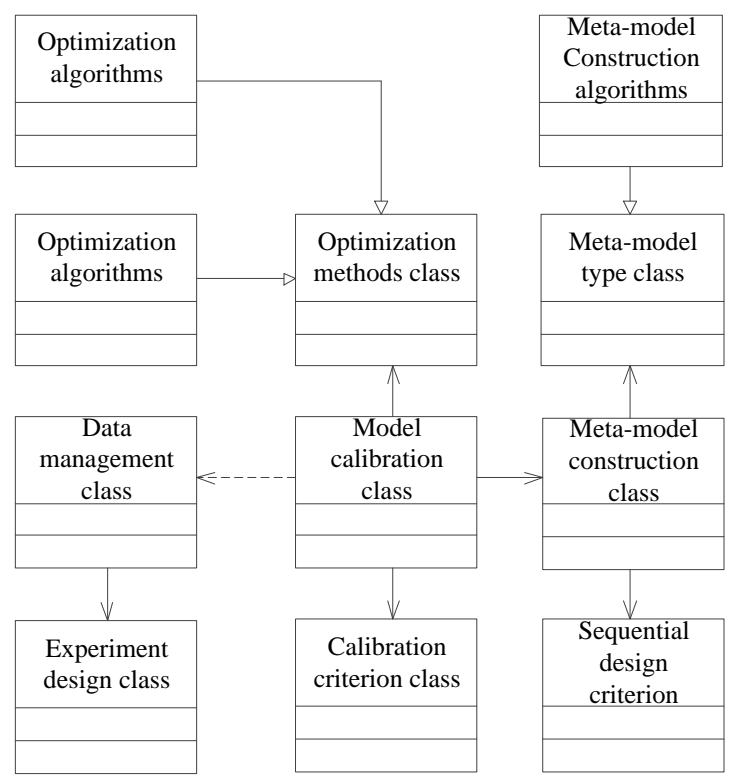

Figure 6. Class diagram of the model calibration subsystem.

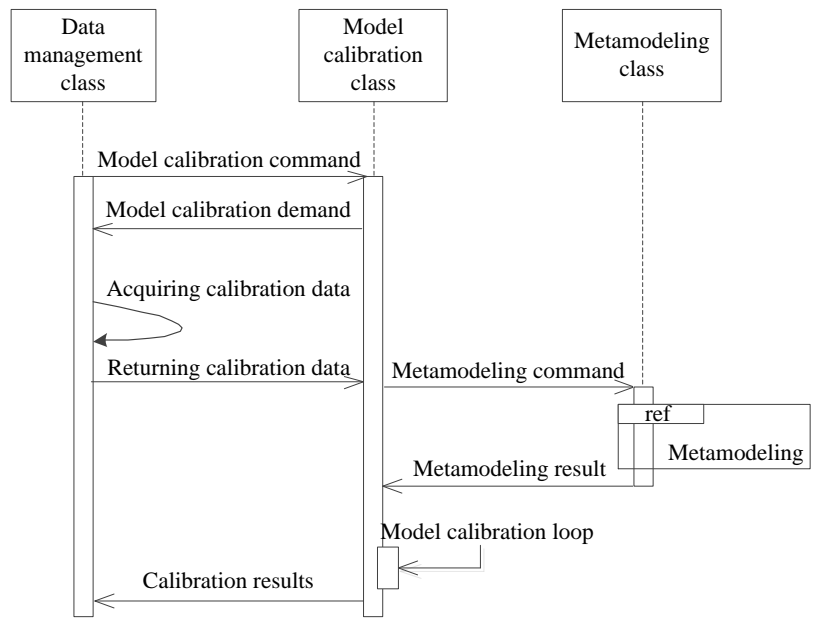

Figure 7. Interactive procedure of model calibration.

After that, the experiment schemes are designed and executed to propagate the uncertainty influence from input parameters to simulation results. LHS method is used to sample the uncertainty parameters. The simulation results could be achieved and used to validate the model credibility with the experimental observations. The result of model validation is given in Figure 10. The blue envelope lines represent the evidence body of model output and the red envelope lines are the evidence body of experiment observations. The evidence distance of two evidence bodies is 
0.6581, which represents the model validation result before calibration.

Finally, the epistemic uncertainty parameters of model need to be calibrated. The consistency metamodel which is obtained through multiple model output and experiment observations is established based on sequential design method in Figure 11. The model calibration information are configured such as optimization methods, terminal conditions etc. After calibrating the epistemic uncertainty parameter, the validation result after calibration is given in Figure 12 and the later evidence distance is 0.9398 . Through comparing the validation result of before and after calibration, the kinematic model becomes more precise and HIT-MVCP can implement validation and calibration of simulation models effectively.

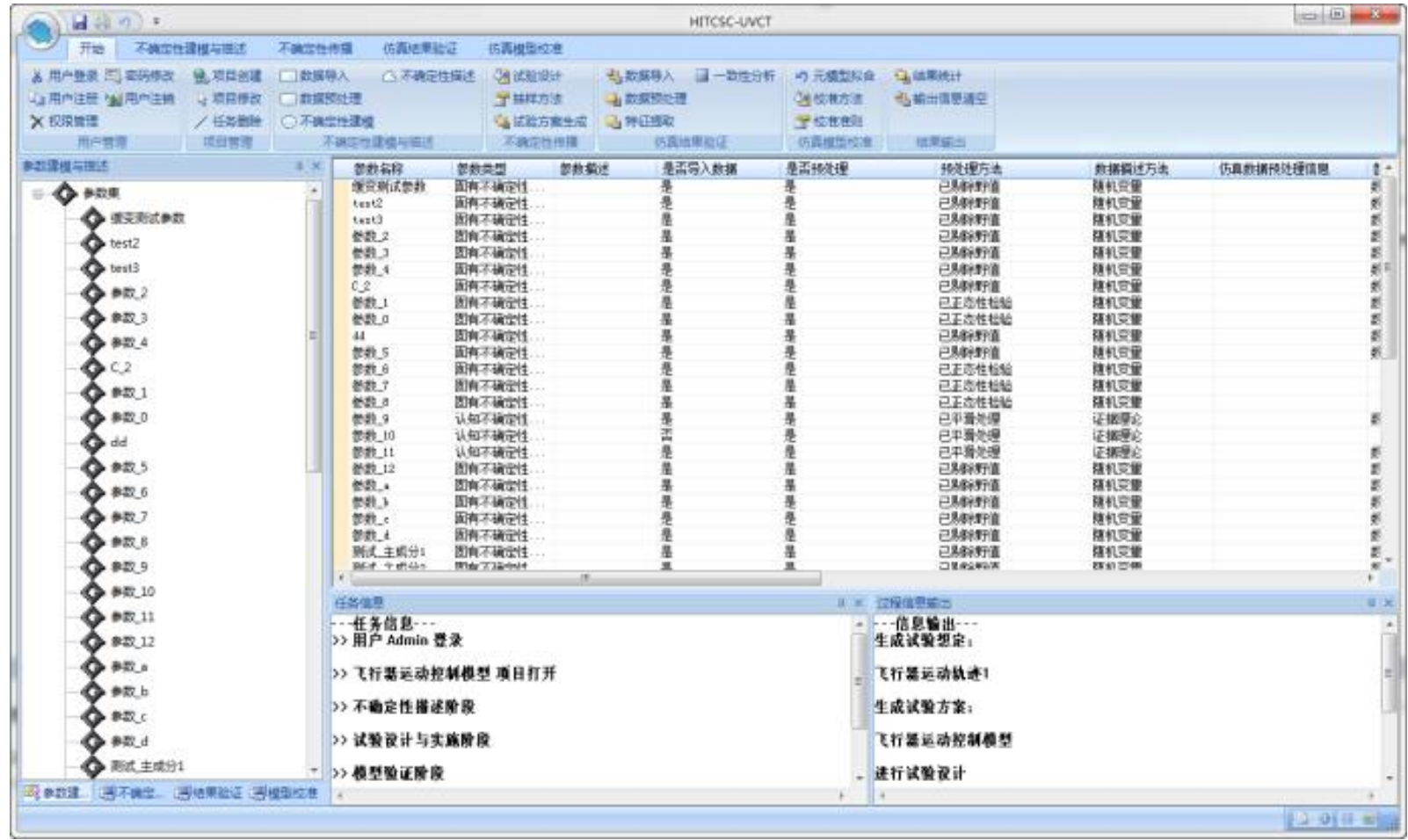

Figure 8. The human-computer interactive interface of HIT-MVCP.

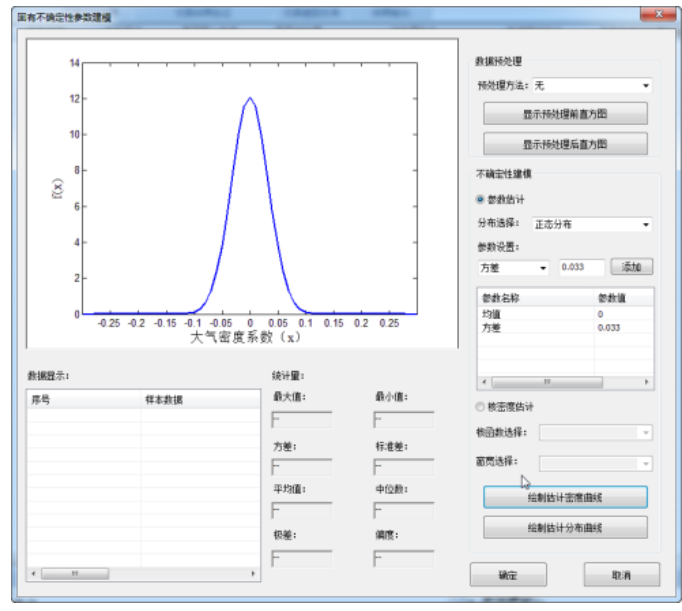

Figure 9. Configuration interface of the uncertainty parameters.

\section{Conclusions}

With the development of modeling and simulation techniques, more and more complex simulation models need to be validated and calibrated. Due to SMVC under uncertainty is a complicated and tedious work, which includes quantification of various uncertainties, design of many simulation experiments, complex data management and analysis etc. So this paper develops an auxiliary SMVC platform, HIT-MVCP. A process model is presented firstly to explicate the operation procedures of SMVC under uncertainty. Then, the structure and function of HIT-MVCP are designed and the software technique of strategy pattern is introduced to design maintainability and extendibility of the SMVC algorithms. Finally, the software implementation of HIT-MVCP is given and an example of model validation and calibration of a flight vehicle kinematic control system is illustrated how to use the platform. This example shows that HIT-MVCP could assist professional to accomplish the SMVC under uncertainty effectively.

Currently, the epistemic uncertainty is only described based on interval theory and this will cause the incomplete description of uncertainty information sometimes. In future work, many other description methods such as evidence theory and imprecise probability theory etc. need to be introduced to describe the uncertainty parameter. Besides, the SMVC algorithms involved in the platform such as methods of 
experiment schemes design, model validation algorithms and model calibration methods etc. are insufficient to adapt to the SMVC of different complex simulation models, so more related algorithms need to be introduced into HIT-MVCP in the future.

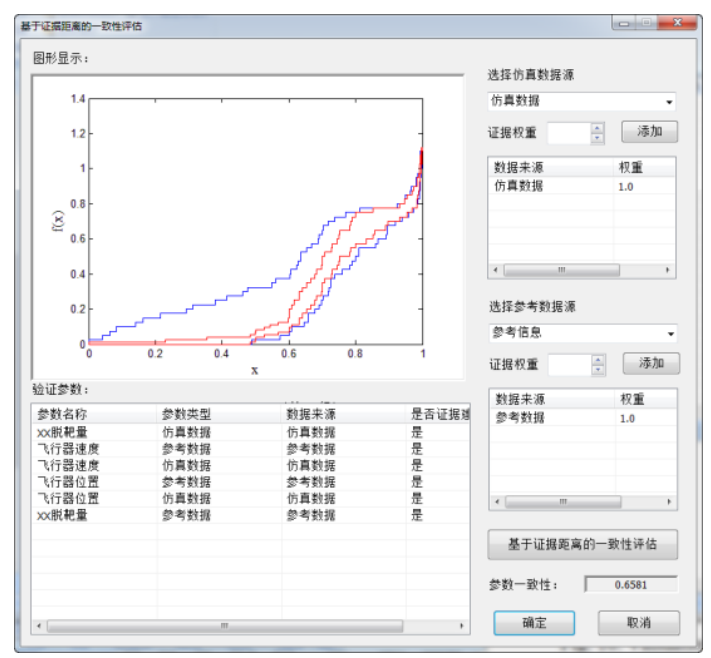

Figure 10. Validation results before calibration.

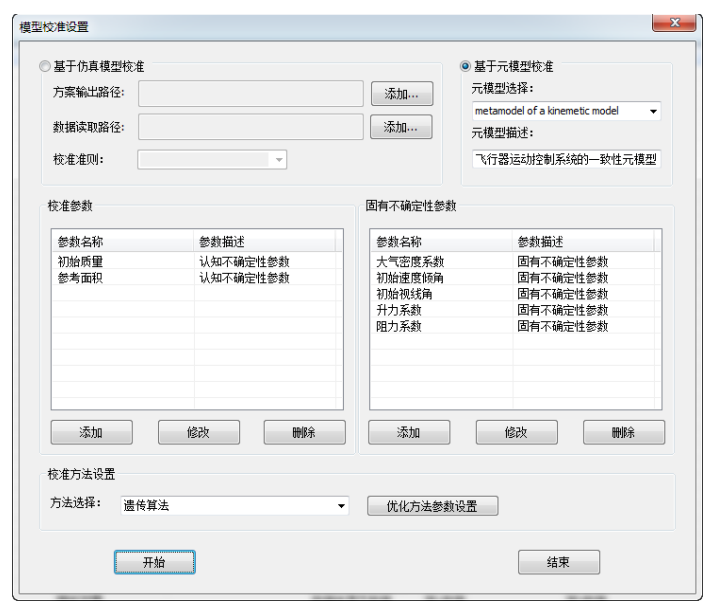

Figure 11. Configuration interface of the model calibration.

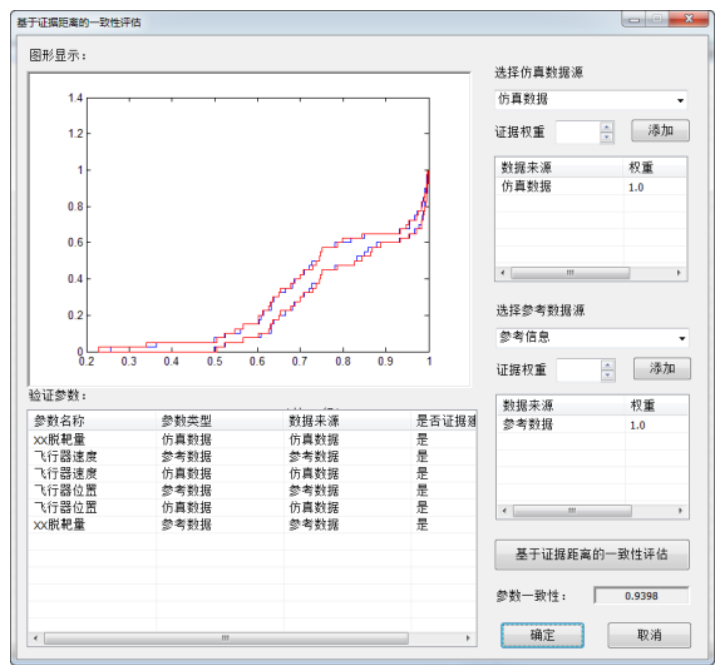

Figure 12. The validation results after calibration.

\section{Acknowledgements}

This research is supported by the National Natural Science Foundation of China (Grant No. 61403097).

\section{References}

O. Balci, R. J. Adams, and D. S. Myers. A Collaborative Evaluation Environment for Credibility Assessment of Modeling and Simulation Applications. Simulation Conference, Proceedings of the Winter. U.S.: San Diego, CA. 214-220, 2002. doi:10.1109/wsc.2002.1172887.

D. Berleant. Automatically Verified Reasoning with both Intervals and Probability Density Functions. Interval Computations, 2:48-70, 1993. doi:10.1007/978-1-46133440-8_10.

S. Ferson. RAMAS Risk Calc 4.0 Software: Risk Assessment with Uncertain Numbers. CRC Press, 2002.

Jon C. Helton. Quantification of Margins and Uncertainties: Conceptual and Computational Basis. Reliability Engeering \& System Safety, 96:976-1013, 2011. doi:10.1016/j.ress.2011.03.017.

Jon C. Helton, J. D. Johnson, and C. J. Sallaberry. Survey of Sampling-based Methods for Uncertainty and Sensitivity Analysis. Reliability Engineering \& System Safety, 91:1175-1209. 2006. doi:10.1016/j.ress.2005.11.017.

L. Meng, X. Qian, and H. Wang. Result Validation of A Rudder Simulation Model. 27th European Modeling and Simulation Symposium. Italy: Bergeggi, 63-69, 2015.

X. Qian, W. Li, and M. Yang. Two-stage Nested Optimization-based Uncertainty Propagation Method for Model Calibration. International Journal of Modeling, Simulation, and Scientific Computing, 7:1-17, 2016. doi:10.1007/978-3-642-45037-2_23.

Christopher J. Roy and William L. Oberkampf. A Comprehensive Framework for Verification, Validation, and Uncertainty Quantification in Scientific Computing. Comput. Methods Appl. Mech. Engrg. 200:2131-2144, 2011. doi:10.1016/j.cma.2011.03.016.

A. Saltelli, S. Tarantola, F. Campolongo, and M. Ratto. Sensitivity Analysis in Practice: A Guide to Assessing Scientific Models. Journal of the Royal Statistical Society. Series A, Statistics in Society, 168:4466-4473, 2005. doi:10.1111/j.1467-985X.2005.358_16.x.

S. Sankararaman and S. Mahadevan. Model Validation under Epistemic Uncertainty. Reliability Engineering and System Safety, $\quad 96: 1232-1241$, 2011. doi:10.1016/j.ress.2010.07.014. 